Invest. pens. crit. (ISSN 1812-3864)

Vol. 5, No. 1, enero-abril 2017

pp. $17-27$

\title{
Percepciones y Prácticas Relacionadas a la Excelencia Educativa en las Escuelas Oficiales de Panamá
}

\section{Dra. Nadia De León}

\author{
Investigadora Asociada \\ INDICASAT \\ nadiadeleonporter@gmail.com
}

Recibido: 16 de marzo de 2017

Aceptado: 17 de abril de 2017

\section{Resumen}

Este estudio se basa en un análisis de correlación entre los resultados del Concurso Nacional por la Excelencia Educativa 2016 provenientes de todo Panamá, y las percepciones y prácticas encontradas en los ensayos y entrevistas presentadas por las escuelas como parte de su aplicación para el concurso. Las escuelas ganadoras fueron aquellas cuyos estudiantes obtuvieron los puntajes más altos en pruebas de lenguaje, ciencias, y matemáticas. Se encontró que las percepciones con un impacto significativo en la calidad educativa incluyen: la percepción de falta de capacitación docente como barrera a la excelencia; y tener un concepto de excelencia educativa que incluye la inclusión, y los logros de estudiantes y egresados. Las siguientes practicas están correlacionadas con puntajes más altos, pero individualmente no llegan a tener un impacto significativo en las pruebas: el acompañamiento pedagógico a los docentes, el uso de métodos de enseñanza activa; participar de ciertos programas de capacitación docente a largo plazo y con seguimiento adicionales a aquellos obligatorios presentados por el Ministerio de Educación (particularmente en más de uno de ellos ya que se dirigen en parte a aspectos diferentes); ejemplos de esfuerzos hacia la excelencia que involucren la evaluación de aprendizajes y el liderazgo, definido como diagnosis de problemas utilizando indicadores, toma de decisiones informadas para implementar intervenciones, y evaluación de impacto de dichas acciones. Las siguientes prácticas están significativa e inversamente relacionadas con la excelencia educativa: haber mencionado ejemplos de esfuerzo hacia la excelencia relacionados a la infraestructura, actividades adicionales, $y / o$ disciplina.

\section{Palabras clave}

Educación, Panamá, percepción, calidad, prácticas, impacto

This study is based on a correlation analysis between the results of the National Competition for Excellence in Education 2016 from all over Panama and the perceptions and practices found in the tests and interviews presented by the schools as part of their application for the competition. The winning schools were those whose students scored the highest in language, science, and math tests. It was found that perceptions with a significant impact on educational quality include: perceived lack of 
Invest. pens. crit. (ISSN 1812-3864)

Vol. 5, No. 1, enero-abril 2017

pp. $17-27$

teacher training as a barrier to excellence and having a concept of educational excellence that includes inclusion, and achievements of students and graduates. The following practices are correlated with higher scores, but individually they do not have a significant impact on the tests: the pedagogical accompaniment to the teachers, the use of active teaching methods; participation in certain long-term teacher training programs with additional follow-up to those mandated by the Ministry of Education (particularly in more than one of them since they address in part to different aspects. Examples of efforts towards excellence that involve evaluation of learning and leadership, defined as diagnosis of problems using indicators, informed decision makers on how to implement interventions and to conduct evaluation of the impact of such actions. The following practices are significantly and inversely related to educational excellence: mentioning examples of effort toward excellence related to infrastructure, additional activities, and/or discipline.

\section{Introducción}

Este análisis se basa en los documentos y resultados obtenidos de las 100 escuelas finalistas en el Concurso Nacional por la Excelencia Educativa 2016, provenientes de todo el país. Las escuelas participaron voluntariamente. El concurso se llevó a cabo en tres fases. En la Fase 1, los participantes presentaron ensayos y participaron en entrevistas. Evaluando dichos ensayos y entrevistas, se escogieron 100 semifinalistas. En la Fase 2, diez estudiantes de cada escuela, escogidos aleatoriamente, tomaron una prueba estandarizada con ítems tipo PISA para medir conocimientos en ciencia, matemática y lenguaje, desarrollada para el concurso por especialistas internacionales específicamente para 6to, 9no, y 12vo grado, dependiendo de la categoría en la que participaba la escuela. En base a los resultados de esa prueba, se escogieron 21 finalistas. En la Fase 3, otra vez, diez estudiantes de cada escuela, escogidos aleatoriamente, tomaron una prueba comparable, y en base a los resultados se escogieron los 9 ganadores: primer lugar, segundo lugar y mención de honor para cada una de las 3 categorías.

\section{Marco teórico}

El consenso en ciencias de la educación es medir la excelencia educativa, o efectividad de las escuelas, basados en el aprendizaje de los estudiantes (Reynolds et al. 2001). Dicho aprendizaje suele ser medido por medio de pruebas internacionales estandarizadas, ya que permiten producir información fácilmente generalizable y comparable, y se enfocan en los tipos de aprendizaje sobre cuya importancia hay común acuerdo de país en país.

Las investigaciones y meta-análisis de investigaciones en el campo de las ciencias de la educación identifican que la variable con mayor impacto en el aprendizaje de los estudiantes son los docentes, seguidos por los directores (Bell, Bolam, and Cubillo, 2003). La infraestructura y el uso de la tecnología tienen poco o ningún impacto en los resultados obtenidos por los estudiantes, más allá de la infraestructura básica como los baños y salones con temperatura cómoda (OECD, 2010; OECD, 2015).

Se han generado muchísimas listas de características de escuelas efectivas, pero podemos resumir y aglomerar los resultados de la siguiente manera: 
Invest. pens. crit. (ISSN 1812-3864)

Vol. 5, No. 1, enero-abril 2017

pp. $17-27$

\section{Liderazgo}

- Buen liderazgo por parte de los administradores a nivel alto y medio con la capacidad de identificar y resolver problemas y facilitar cambios (Smith and Tomlinson, 1990; Department of Education and Science, 1988)

- Una organización y gerencia simple, clara y lo más plana posible, con participación de los docentes en procesos de auto-evaluación constantes y toma de decisiones (Smith and Tomlinson, 1990; Brighouse and Tomlinson, 1991: 5; Sammons et al., 1995; Reynolds et al., 1996)

- Objetivos claros y compartidos, traducidos a prácticas pedagógicas con énfasis en estándares académicos altos (Department of Education and Science, 1988; Sammons et al., 1995; Reynolds et al., 1996; Reynolds,1995)

- Espíritu de la escuela con un buen ambiente de trabajo y respeto y altas expectativas para los estudiantes y el personal (Smith and Tomlinson, 1990; Rutter et al., 1979; Brighouse and Tomlinson, 1991: 5; Sammons et al., 1995; Reynolds et al., 1996)

- Un ambiente positivo, ordenado y estimulante con manejo efectivo de los estudiantes en el salón de clases(Department of Education and Science, 1988; Rutter et al. 1979; Sammons et al., 1995; Reynolds et al., 1996)

\section{Docentes}

- Docentes bien capacitados con conocimiento pedagógico y de los temas que enseñan, y consistencia a lo largo de los años (Department of Education and Science, 1988; Reynolds,1995)

- Desarrollo profesional constante para el personal (Brighouse and Tomlinson, 1991: 5)

- Docentes que son modelos positivos a seguir para los estudiantes (Rutter et al., 1979)

- Trato y realimentación positiva a los estudiantes con seguimiento a su bienestar integral, relaciones positivas y apoyo a su individualidad (Smith and Tomlinson, 1990; Department of Education and Science, 1988; Rutter et al., 1979; Sammons et al., 1995; Reynolds et al., 1996)

\section{Enseñanza-Aprendizaje}

- Utilización de métodos de enseñanza efectivos, estructurados y adaptables (Sammons et al., 1995; Reynolds et al., 1996)

- Un currículo bien planeado, y con consistencia entre grados y materias (Department of Education and Science, 1988; Reynolds,1995)

Comunidad

- Relaciones positivas con la comunidad e involucración de los padres (Department of Education and Science, 1988; Brighouse and Tomlinson, 1991: 5; Sammons et al., 1995; Reynolds et al., 1996; Reynolds,1995)

Para el contexto especifico de países en desarrollo, se ha encontrado que los factores asociados con los logros de los estudiantes también incluyen: el tiempo de instrucción, actividad en la biblioteca de la escuela, años de formación de los docentes, y los libros y materiales educativos usados (Reynolds et 
Invest. pens. crit. (ISSN 1812-3864)

Vol. 5, No. 1, enero-abril 2017

pp. $17-27$

al., 2000: 240). En referencia a sistemas educativos, también sabemos que en los países con éxito educativo las escuelas tienden a tener mayor autonomía (OECD, 2015).

\section{METODOLOGÍA}

Este estudio se llevó a cabo utilizando los resultados de un estudio previo que consistió en un análisis cualitativo de los ensayos y entrevistas que formaron parte del proceso de aplicación de cada escuela para participar en el concurso. El estudio utilizó codificación por temas, incluyendo percepciones sobre la definición de excelencia educativa y lo que hace falta en cada escuela para lograrla, así como ejemplos de esfuerzos y logros en la calidad educativa. Los resultados permitieron saber cuáles escuelas presentaban algunas de las 212 percepciones y prácticas codificadas. Para este estudio se llevó a cabo análisis cuantitativo que consistió en explorar la correlación de dichas percepciones y prácticas con los resultados del concurso y los puntajes obtenidos por los estudiantes de dichas escuelas en las pruebas del concurso, como medidas de excelencia educativa. Este análisis consiste en dos partes, un análisis de frecuencia de factores predictivos entre los semifinalistas, finalistas y ganadores; y un análisis de correlación significativa entre los códigos y los puntajes obtenidos por cada escuela.

\section{Resultados}

\section{Predictores de éxito en las pruebas}

Para evaluar el impacto de las variables identificadas por medio del análisis cualitativo de los ensayos y entrevistas en el éxito que tuvo cada escuela en el concurso, utilizamos varios métodos de análisis. Primero se utilizó one-way between-subjects MANOVA con los 212 códigos como variables independientes y dos variables dependientes: 1) el puntaje obtenido en la prueba de la Fase 2, y 2) el puntaje obtenido en la prueba de la Fase 3. Se encontró un impacto significativo (positivo o negativo) de las siguientes variables en los resultados de las pruebas $(\mathrm{p}<0.05)$ :

- Haber mencionado actividades adicionales ofrecidas a los estudiantes (curriculares o extracurriculares) como ejemplos de acciones específicas tomadas para mejorar la calidad educativa durante la entrevista

- Haber mencionado participación en las capacitaciones docentes ofrecida por el Ministerio de Educación ya sea en la entrevista o ensayo

- Haber mencionado la participación de estudiantes en concursos como parte de la definición de excelencia en el ensayo

- Haber mencionado la inclusión como parte de la definición de excelencia en el ensayo.

- Haber mencionado el liderazgo como parte de la definición de excelencia en el ensayo.

- Haber mencionado a los egresados de la escuela ya sea en el ensayo o la entrevista.

- Haber mencionado la participación en la jornada extendida como ejemplo de esfuerzo hacia la excelencia.

- Haber mencionado el acompañamiento pedagógico a la labor del docente Como ejemplo de esfuerzo hacia la excelencia

- Haber mencionado esfuerzos hacia la excelencia relacionados a la infraestructura y materiales en el ensayo

- Haber mencionado la falta de capacitación docente como barrera a la excelencia Durante la entrevista 
Invest. pens. crit. (ISSN 1812-3864)

Vol. 5, No. 1, enero-abril 2017

pp. $17-27$

Como segundo método se utilizó one-way between-subjects ANOVA con los 212 códigos como

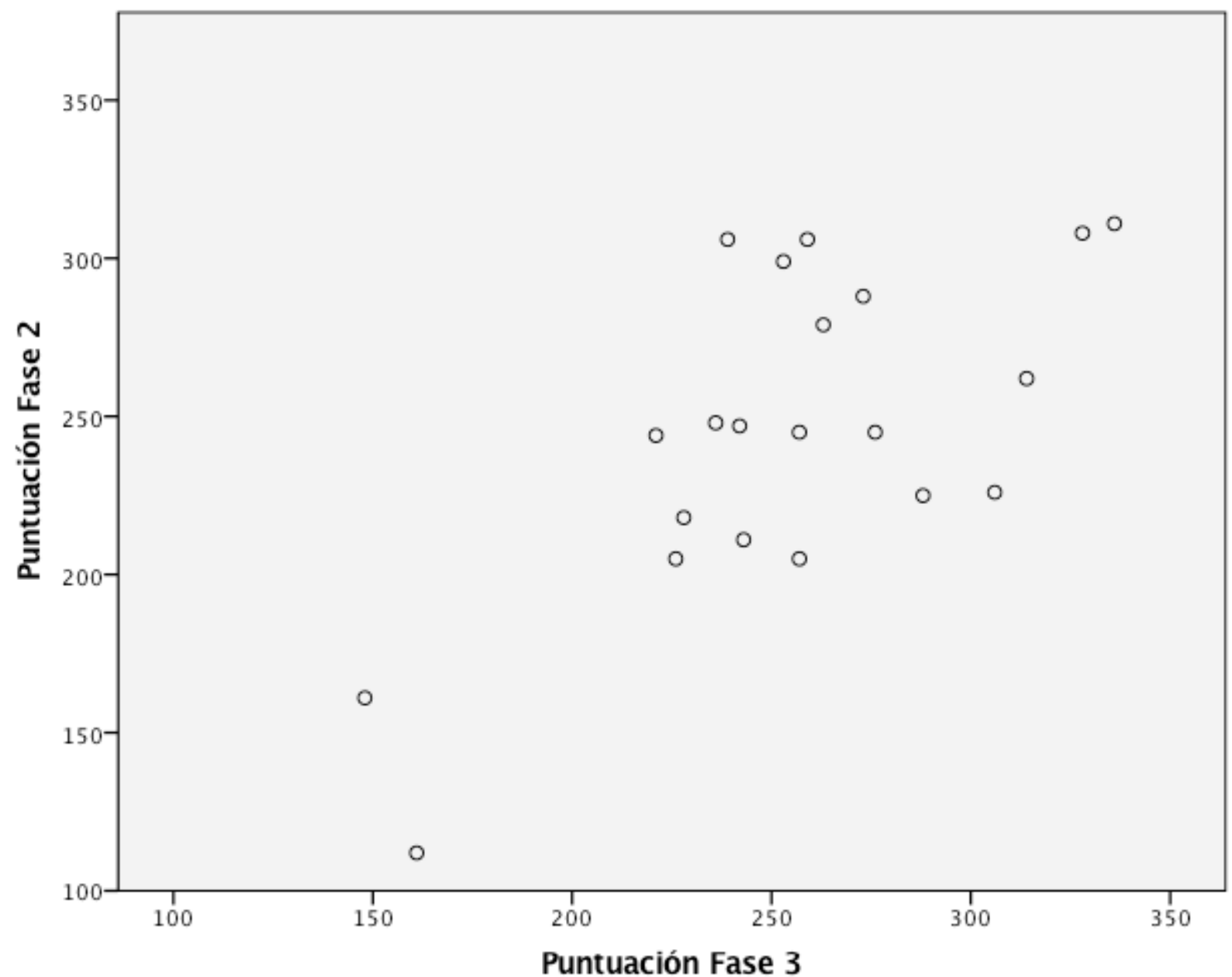

variables independientes y el puntaje obtenido en la prueba de la Fase 2 como variable dependiente, para analizar el impacto en esta prueba por separado. Se encontró un impacto significativo de las mismas variables excepto por acompañamiento pedagógico $(\mathrm{p}<0.05)$.

El limitado tamaño de la muestra de escuelas que tomaron la prueba de la Fase 3 dificultó el análisis del impacto de estas variables en esta prueba por separado. Sin embargo, se realizó un análisis de correlación entre los puntajes obtenidos en la Fase 2 y en la Fase 3 para las escuelas que tomaron ambas pruebas. Para ello se computó el coeficiente de correlación de Pearson's y se encontró una correlación significativa $(\mathrm{r}=0.691, \mathrm{n}=21, \mathrm{p}=0.001)$, lo cual refuerza la confiabilidad de la prueba $\mathrm{y}$ nos lleva a escoger basarnos en MANOVA usando ambas pruebas y ANOVA sólo con la prueba de la Fase 2, sin aislar el impacto en la prueba de la Fase 3.

Para crear una modelo de características que predigan éxito en la prueba, se inició por tomar las variables con correlación significativa en los resultados de al menos uno de los dos análisis descritos en la sección anterior. A la lista le agregamos las siguientes variables que consideramos como posibles predictores de éxito, ya que la literatura en ciencias de la educación indica que son características 
Invest. pens. crit. (ISSN 1812-3864)

Vol. 5, No. 1, enero-abril 2017

pp. $17-27$

escolares que llevan a la excelencia educativa, la experiencia del autor dentro del contexto Panameño le indican su relevancia, son características que aparecen en el $20 \%$ o menos de los ensayos y entrevistas (lo cual les da más poder de predicción), y/o tuvieron una correlación relevante (positiva o negativa) con el puntaje otorgado por los evaluadores en la Fase 1:

- Haber mencionado la participación en el programa Panamá Bilingüe del Ministerio de Educación como ejemplo de esfuerzo hacia la excelencia

- Haber mencionado la participación de los estudiantes en actividades adicionales curriculares

- Haber mencionado la participación de docentes en capacitaciones

- Haber mencionado la participación de docentes en comunidades de aprendizaje profesional

- Haber mencionado métodos de enseñanza activa como ejemplos de esfuerzo hacia la excelencia

- Haber mencionado la evaluación ya sea en definición de excelencia, percepción de lo que falta para alcanzar la excelencia, o esfuerzo hacia la excelencia.

- Haber mencionado el liderazgo ya sea en definición de excelencia, percepción de lo que falta para alcanzar la excelencia, o esfuerzo hacia la excelencia.

- Haber mencionado la disciplina ya sea en definición de excelencia, percepción de lo que falta para alcanzar la excelencia, o esfuerzo hacia la excelencia.

Todas las variables del modelo final están positiva y directamente correlacionadas con los resultados de las pruebas, excepto las siguientes variables que están inversamente correlacionados:

- Haber mencionado la participación del estudiante en actividades adicionales como ejemplo de esfuerzo hacia la excelencia

- Haber mencionado esfuerzo relacionados a la infraestructura y materiales como ejemplo de fuerzas en la excelencia

- Haber mencionado la disciplina ya sea en definición de excelencia, percepción de lo que falta para alcanzar la excelencia, o esfuerzo hacia la excelencia.

Finalmente, se le adjudicó una calificación predictiva a cada escuela calculada sumando el número de variables predictivas directamente correlacionadas menos aquellas inversamente correlacionadas. Para comprobar la validez del modelo se utilizó Pearson's correlation y se encontró una correlación significativa entre calificación predictiva y puntaje en la prueba de la Fase $2(\mathrm{r}=.203, \mathrm{n}=100, \mathrm{p}=$ 0.043),

\section{Relación entre predictores y éxito}

Para fines de esta discusión nos referimos a las escuelas ganadoras (9), las escuelas finalistas (21), las escuelas semifinalistas (100) y a las escuelas que no tuvieron éxito (105). Definimos como escuelas con éxito a las 21 escuelas que llegaron a ser finalistas.

En general, no hubo relación entre tener una característica predictiva y llegar a ser finalista o ganador. Sólo un centro sin predictores llegó a ser finalista y sólo un centro con un sólo predicar llegó a ser semifinalista. Los predictores normalmente sólo se volvieron efectivos al acumularse dos o más; con excepción de actividades adicionales curriculares, única característica predictiva presente en el centro que llegó a ser semifinalista con un solo predictor. Todos los centros ganadores tenían 2 o más predictores. 
Invest. pens. crit. (ISSN 1812-3864)

Vol. 5, No. 1, enero-abril 2017

pp. $17-27$

La característica más común entre las escuelas con éxito es la presencia de actividades adicionales $(86 \%$ de las escuelas finalistas). Sin embargo, ésta es también una característica común entre las escuela que no tuvieron éxito $(72 \%)$. La segunda característica más común entre las escuelas con éxito es la presencia de actividades adicionales específicamente curriculares (es decir, relacionadas a lenguaje, matemáticas y ciencia; y no actividades extracurriculares como deportes, religión, o folklore) (62\% de las escuelas semifinalistas). Sin embargo, esta es también una característica común entre las escuelas que no tuvieron éxito ( $74 \%$ de las escuelas que no tuvieron éxito). De hecho, como observamos, las escuelas con éxito presentaron menos actividades adicionales curriculares que las escuelas sin éxito, contrario a lo teorizado por los investigadores. Es importante recordar, incluso, que haber mencionado este tipo de actividades de cualquier tipo como ejemplos de excelencia o acciones hacia la excelencia está inversamente relacionado con los puntajes en las pruebas de la Fase 2.

Las diferencias notables entre las escuelas con éxito y las escuelas sin éxito (diferencia mayor al 5\%) son:

- Que hayan mencionado a sus egresados como ejemplos de excelencia (43\% de las escuelas con éxito vs $11 \%$ de las escuelas sin éxito)

- Que haya mencionado el liderazgo como parte de la definición de excelencia educativa (19\% de las escuelas con éxito vs 6\% de las escuelas sin éxito).

- Que hayan mencionado la falta de capacitación docente (14\% de las escuelas con éxito, versus 4\% de las escuelas sin éxito).

- Que hayan mencionado el ambiente en la escuela, tanto laboral como para los estudiantes, como parte de la definición de excelencia educativa (19\% en las escuelas con éxito vs $9 \%$ en las escuelas sin éxito)

- Que hayan mencionado la enseñanza activa y otras pedagogías relacionadas como ejemplos de excelencia o acciones tomadas para mejorar la calidad de la educación (14\% de las escuelas con éxito vs $8 \%$ de las escuelas sin éxito)

- Que hayan mencionado el acompañamiento pedagógico al docente por parte del director y/o supervisor como ejemplo de excelencia o acciones tomadas para mejorar la calidad de la educación (10\% de las escuelas con éxito vs $2 \%$ de las escuelas sin éxito)

- Que hayan mencionado motivar a y/o aprender de otros como parte de su propósito para participar en el concurso (10\% de las escuelas con éxito vs 3\% de las escuelas sin éxito)

Notamos también que ninguna escuela que haya mencionado la disciplina llegó a ser finalista. De hecho, recordemos que esta mención está inversamente relacionada con la puntuación en la fase 2. De la misma manera, llama la atención que, contrario a lo teorizado por los investigadores, menos escuelas con éxitos mencionaron la evaluación que escuelas sin éxito (14\% vs 16\%). También es importante resaltar que características calificadas durante la entrevista como el interés alto en participar en el concurso, la coherencia entre ellos y con el ensayo, y el trabajo en equipo no resultaron predictores de éxito.

Examinamos en más detalle también la participación en tres programas de capacitación docente sobrerepresentados entre los finalistas. Los tres programas son capacitaciones a docentes a largo plazo y con seguimiento individualizado a cargo de facilitadores, y no talleres que ocurren una sola vez. De entre las escuelas semifinalistas en el concurso, el 25\% participaron de al menos uno de estos tres 
Invest. pens. crit. (ISSN 1812-3864)

Vol. 5, No. 1, enero-abril 2017

pp. $17-27$

programas. Sin embargo, el $71 \%$ de las escuelas finalistas y el $78 \%$ de las escuelas ganadoras participaron en estos programas (y esto tomando en cuenta que algunos de los centros con éxito se encuentran fuera de las áreas de cobertura geográfica de algunos de dichos programas). De hecho, cinco de las veintiuna escuelas finalistas participaron en dos de estos programas a la vez, y las cinco resultaron ganadoras. Para el caso de cada uno de estos tres programas, las escuelas participantes lograron puntajes más altos tanto en las pruebas de la Fase 2 como en las pruebas de la Fase 3. Sin embargo, para ninguno de los 3 programas dichas diferencias fueron significativas. La siguiente tabla presenta los detalles:

\begin{tabular}{|l|l|l|l|l|}
\hline Programa & $\begin{array}{l}\text { Diferencia en } \\
\text { puntos entre } \\
\text { participantes y no } \\
\text { participantes en } \\
\text { pruebas Fase 2 }\end{array}$ & $\begin{array}{l}\text { Resultados de } \\
\text { ANOVA }\end{array}$ & $\begin{array}{l}\text { Diferencia en } \\
\text { puntos entre } \\
\text { participantes y no } \\
\text { participantes en } \\
\text { pruebas Fase 3 }\end{array}$ & $\begin{array}{l}\text { Resultados de } \\
\text { ANOVA }\end{array}$ \\
\hline Programa 1 & 13 & $\begin{array}{l}\mathrm{F}(1,98)=.513, \mathrm{p}= \\
.476\end{array}$ & 25 & $\begin{array}{l}\mathrm{F}(1,19)=1.358, \mathrm{p} \\
=.258\end{array}$ \\
\hline Programa 2 & 28 & $\begin{array}{l}\mathrm{F}(1,98)=.976, \mathrm{p}= \\
.326\end{array}$ & $\begin{array}{l}\mathrm{F}(1,19)=.236, \mathrm{p}= \\
.633\end{array}$ \\
\hline Programa 3 & 16 & $\begin{array}{l}\mathrm{F}(1,98)=.469, \mathrm{p}= \\
.495\end{array}$ & $\begin{array}{l}\mathrm{F}(1,19)=1.049, \mathrm{p} \\
=.319\end{array}$ \\
\hline
\end{tabular}

\section{Discusión \\ Patrones}

\section{Docentes, Procesos de Enseñanza-Aprendizaje, y Educación Integral y en Valores}

En concordancia con lo que indican los estudios a la fecha en ciencias de la educación, una de las variable importantes entre las escuelas con éxito parece ser la capacitación docente, en particular ciertas capacitaciones de calidad adicionales a las presentadas por MEDUCA para todas las escuelas. A luz de esto, resulta preocupante la bajísima proporción de escuelas que mencionan la falta de capacitación docente como una barrera a la excelencia (7\%). También preocupa que no parece haber comprensión entre los directos de los programas de capacitación docente de ProEd y Hagamos Ciencia como tales. Sin embargo, por el otro lado, también parece que dichos programas están teniendo un impacto en los docentes relacionado al éxito en el concurso, a pesar de la falta de comprensión y/o apoyo de los directores. El impacto, menor del esperado de acuerdo al marco teórico, de las capacitaciones docentes también parece indicar un problema de baja calidad en las capacitaciones que reciben nuestros docentes.

Preocupa también el bajísimo nivel de ejemplos y definiciones relacionadas a la utilización de pedagogías efectivas por parte de los docentes, y la total ausencia de respuestas por partes de las escuelas que reconozcan que la mayoría de los docentes no utilizan éstos métodos efectivamente como 
Invest. pens. crit. (ISSN 1812-3864)

Vol. 5, No. 1, enero-abril 2017

pp. $17-27$

algo que les falta para llegar a la excelencia. Estos resultados comprueban lo indicado por los estudios en el tema: el factor más importante en la excelencia educativa son los docentes y la calidad de trabajo, que sólo puede ser proporcional a su formación inicial y continúa.

\section{Directores, Acompañamiento Pedagógico, Liderazgo y Evaluación}

Aparte de lo relacionado a la capacitación docente, los resultados también parecen confirmar la importancia de ciertas características lamentablemente poco comunes en nuestras escuelas (presentes en menos de un cuarto de las escuelas y en algunos casos en menos del 10\%) pero correlacionadas con éxito. Los resultados, sin embargo, nos demuestran que la presencia de solo una de estas características no es suficiente para lograr el éxito, sino que su impacto aumenta aceleradamente y es sólo significativo una vez que se combinan dos o más de dichas características. Estas características incluyen todos los aspectos del rol del director: acompañamiento pedagógico de los docentes, mejoras institucionales, liderazgo, y evaluación. Estos resultados comprueban lo indicado por los estudios en el tema: el segundo factor más importante en la excelencia educativa son los directores. Tomando esto en consideración, preocupa la falta de evidencia de evaluación y liderazgo en los ensayos y entrevistas, así como la superficialidad de los ejemplos mencionados que demuestran poca capacidad de utilizar evidencias, diagnosticar dificultades, o medir éxito utilizando indicadores; aparte del bajo nivel de los ensayos escritos, muchos de los cuales fueron redactados o revisados por los directores de los centros.

Los patrones más comunes no son los de mayor impacto

Las investigaciones en ciencias de la educación han demostrado el bajo impacto de la infraestructura y tecnología en la calidad de la educación, más allá de un nivel mínimo requerido, es lamentable que sea el patrón más alto encontrado en los ensayos y entrevistas. De hecho, su correlación inversa con los resultados de las pruebas en Fase 2 refuerza las conclusiones científicas internacionales en un contexto local.

De la misma manera, preocupa altamente el hecho de que las actividades adicionales estén también inversamente correlacionadas con los resultados de las pruebas y/o el éxito en el concurso, incluso aquellas actividades consideradas como curriculares. Los investigadores teorizan que la única manera de explicar este hallazgo es como evidencia de la bajísima calidad de dichas actividades.

\section{Buenas Prácticas}

Las prácticas con un impacto significativo en haber logrado éxito en este concurso, incluyen:

- haber mencionado la capacitación docente en el ensayo o la entrevista, particularmente la falta de capacitación docente

- tener un concepto de excelencia educativa que incluye la inclusión, y los logros de estudiantes y egresados

Las siguientes prácticas están apoyadas por estudios a la fecha y correlacionados con éxito en el concurso, pero al realizar análisis de correlación de Pearson individualmente, no llegaron a tener un impacto significativo:

- el acompañamiento pedagógico a los docentes 
Invest. pens. crit. (ISSN 1812-3864)

Vol. 5, No. 1, enero-abril 2017

pp. $17-27$

- el uso de métodos de enseñanza activa

- y participar de ciertos programas de capacitación docente a largo plazo y con seguimiento adicionales a aquellos obligatorios presentados por el Ministerio de Educación., y particularmente en más de uno de ellos ya que se dirigen en parte a aspectos diferentes

- las actividades adicionales curriculares o de refuerzo académico para los estudiantes

- ejemplos de esfuerzos hacia la excelencia que involucren la evaluación de aprendizajes y el liderazgo, definido como diagnosis de problemas utilizando indicadores, toma de decisiones informadas para implementar intervenciones, y evaluación de impacto de dichas acciones

- haber mencionado la jornada extendida o participar en Panamá Bilingüe en el ensayo o la entrevista

Las siguientes prácticas están significativamente relacionadas con NO haber tenido éxito en el concurso:

- haber mencionado ejemplos de esfuerzo hacia la excelencia relacionados a la infraestructura

- haber mencionado actividades adicionales como ejemplos de esfuerzos hacia la excelencia educativa

- haber mencionado ejemplos de esfuerzo hacia la excelencia relacionados a la disciplina

\section{Recomendaciones}

Los resultados de esta investigación nos sugieren que las siguientes serían formas efectivas de ayudar a mejorar la calidad de la educación en Panamá, o al menos en estas escuelas:

- Crear conciencia sobre la falta de evaluación confiable de los aprendizajes de los estudiantes

- Apoyar a la generación de métodos para evaluar los aprendizajes de los estudiantes

- Crear capacidad entre los docentes para la implementación de pedagogías efectivas

- Crear capacidad entre los directores, supervisores y administradores públicos de la educación para el liderazgo y la evaluación

- Apoyar el fortalecimiento de programas de capacitación docentes y actividades adicionales para estudiantes con impacto positivo demostrado en los logros de aprendizaje de los estudiantes y/o apoyados por los estudios en ciencias de la educación

- Maximizar la colaboración entre esfuerzos efectivos existentes, especialmente aquellos que están dirigidos a aspectos complementarios

- Minimizar la generación, continuación o dedicación de tiempo a esfuerzos que no tengan un impacto positivo en los logros de aprendizaje de los estudiantes (o peor aún, tengan un impacto negativo)

Los resultados de esta investigación sugieren la importancia de estudiar a mayor profundidad:

- El impacto de programas como Panamá Bilingüe y la jornada extendida, y otros esfuerzos presentes gubernamentales y de ONGs, en los logros académicos de los estudiantes

- El tipo de actividades adicionales, curriculares y extra-curriculares, y concursos en los que se involucran los estudiantes, así como las capacitaciones en las que participan los docentes, para entender las características de las opciones presentes y su uso, así como evaluar confiablemente su efectividad por medio del impacto en las acciones de los docentes y el aprendizaje de los estudiantes 
Invest. pens. crit. (ISSN 1812-3864)

Vol. 5, No. 1, enero-abril 2017

pp. $17-27$

\section{Referencias}

Bell L, Bolam R, Cubillo L (2003) A systematic review of the impact of school leadership and management on student outcomes. In: Research Evidence in Education Library. London: EPPI-Centre, Social Science Research Unit, Institute of Education, University of London.

Brighouse, T. and Tomlinson, J. (1991) Successful Schools. London: Institute of Public Policy Research.

Department for Education and Employment (DfEE) (2000) National Standards for Headteachers. London: DfEE.

OECD (2015), Students, Computers and Learning: Making the Connection, OECD Publishing, Paris.

OECD (2013), PISA 2012 Results: What Makes Schools Successful. OECD Publishing, Paris

OECD (2010), PISA 2009 Results: What Makes Schools Successful. OECD Publishing, Paris

Reynolds, D. (1991) School effectiveness in secondary schools: research and its policy implications. In S. Riddell and S. Brown (eds) School Effectiveness Research: Its Messages for School Improvement. Edinburgh: HMSO.

Reynolds, D. (1995) The effective school: an inaugural lecture. Evaluation and Research in Education, 9 (2), 57-73.

Reynolds, D. (2000) School effectiveness: the international dimension. In C. Teddlie and D. Reynolds (eds) The International Handbook of School Effectiveness Research. London: RoutledgeFalmer.

Reynolds, D., Sammons, P., Stoll, L., Barber, M. and Hillman, J. (1996) School effectiveness and school improvement in the United Kingdom. School Effectiveness and School Improvement, 7 (2), 133-58.

Reynolds, D., Teddlie, C., Creemers, B., Scheerens, J. and Townsend, T. (2000) An introduction to school effectiveness research. In C. Teddlie and D. Reynolds (eds) The International Handbook of School Effectiveness Research. London: RoutledgeFalmer.

Reynolds, D., Hopkins, D., Potter, D., and Chapman, C. (2001) School Improvement for Schools Facing Challenging Circumstances. London: DES.

Sammons, P. (1994) Findings from school effectiveness research: some implications for improving the quality of schools. In P. Ribbins and E. Burridge (eds), op. cit.

Sammons, P., Hillman, J. and Mortimore, P. (1995) Key Characteristics of Effective Schools: a Review of School Effectiveness Research. Report by the Institute of Education, University of London, for the Office for Standards in Education. 